\title{
Collaborative Faculty Professional Development: Bringing the Classroom to the Screen
}

\author{
Deborah Eldridge \\ Western Governors University \\ Rebecca Watts \\ Western Governors University \\ G. Michael Guy \\ City University of New York \\ Ernest Ialongo \\ City University of New York \\ Lucinda Zoe \\ City University of New York
}

When the higher education practice of face-to-face instruction was disrupted by the COVID pandemic, faculty unaccustomed to and/or uncomfortable with online teaching needed to adapt quickly to serve their students. Fortunately, there are faculty and institutions with long histories of online teaching with much to share about the why and how of offering high-quality, deeply engaging digital learning experiences to support the success of higher education students. The paper explores the collaboration of two universities to create professional development delivered through a virtual workshop series to support faculty needs and encourage an emerging community of practice related to online teaching and learning, jointly envisioned and delivered with pilot funding from the Bill and Melinda Gates Foundation. Four key issues conclude the paper: (1) how can success be measured and supported, (2) how can emerging practices be disseminated beyond workshop participants, (3) how can we respond to the need for changes in how we recognize, incentivize, and reward good teaching, and (4) how do we move forward from here?

Keywords: faculty development, online pedagogy, student engagement, virtual learning, teaching excellence, COVID

\section{INTRODUCTION}

In March 2020, as the crisis of the global COVID pandemic deepened, colleges and universities across the world were forced to pivot rapidly to online teaching and learning. At that time, the City University of 
New York (CUNY) shifted all courses across its 25 institutions to online learning, affecting more than 275,000 students and more than 15,000 full- and part-time faculty.

As it became clear that a return to classroom settings would not come quickly, CUNY leaders designed several multi-campus professional development opportunities in online teaching strategies to be offered over the summer months. One such series was a collaborative opportunity that is detailed in this manuscript. The series was well-received with participants indicating interest in continued offerings.

Philanthropic organizations responded quickly as well. In May 2020, the Bill and Melinda Gates Foundation provided funding support to Western Governors University (WGU), an accredited, online, competency-based university, to develop a strategy to deliver educational value for student in the midst of the pandemic.

In partial fulfillment of the scope of work in the grant, WGU partnered with CUNY to connect WGU's expertise in online teaching and learning to design and deliver an interactive series for CUNY faculty focused on student engagement and success. The series, Helping Bring the Classroom to the Screen: Faculty Support for Course Design and Student Engagement in the Online World was scheduled for CUNY's Fall 2021 academic term.

\section{THEORETICAL FRAMEWORK}

\section{For Online Education}

To begin envisioning useful professional development and drafting the content for a series of workshops for faculty amidst a global pandemic, the series developers referenced the scholarly literature in online education and online professional development. From a theoretical framework for online education (Picciano, 2017), a common language of the elements of online education guided conversations in the early stages of development.

As a way to characterize online education in all its possible forms, Picciano (2017) reviewed general and online-specific learning theories as well as the elements of effective online learning environments to propose a multi-modal framework for online education. Revolving around a learning community, Picciano proposed seven elements that interact, wholly or in part, depending upon the course structure (distance learning; teacher-led fully online; hybrid; etc.). The seven elements of online education within a learning community are:

1. Content (platform, media, games)

2. Social emotional (face-to-face teaching, tutoring, advisement)

3. Self-paced independent study (adaptive software)

4. Dialectal/Questioning (discussion board)

5. Evaluation/assessment (assignments, learning analytics)

6. Collaboration/Student generated content/peer review (wiki, mobile tech)

7. Reflection (blog, journal)

The seven elements of the multi-modal framework accommodate a variety of approaches to online education. For example, the framework contextualized WGU's competency-based approach to online education with its emphasis on the elements of content, social/emotional connection through mentoring and course instructor contacts, self-pacing, and evaluation/ assessment of competency. In contrast, CUNY campuses' adoption of either a fully online or hybridized approach were also interpretable through this model. A traditional instructor-led, fully asynchronous course might capitalize on self-pacing but may sacrifice the social-emotional element. A hybridized course that blends asynchronous with synchronous sessions might incorporate many of the elements of a fully online teacher-led course but limit self-pacing to the instructor's tempo.

\section{Implications for PD Design}

Picciano's multi-modal framework for online education laid the groundwork for conceptualizing the themes of PD opportunities to enhance the CUNY faculty's online teaching and their students' learning in 
its varied forms of delivery. Leveraging WGU's online expertise and emphasizing the needs of the CUNY faculty led to the development of a series of PD modules with content incorporating multi-modal elements:

- Content element $=$ Module on design principles

- Dialectic/questioning, collaboration, and reflection elements = Module on instructional techniques

- Social-emotional and self-paced elements = Module on student engagement strategies

In the context of a relationship between CUNY and WGU, the utilization of the Picciano model acknowledged CUNY faculty thought leadership in online education and established a common language for the collaboration. Visualizing the online education elements as circling a learning community was also a useful construct for exploring the student support structures and faculty development opportunities that lead to success in online teaching and learning.

\section{For Professional Development in Online Teaching}

To enhance the understanding of professional development in the online teaching space, Baran, Correia, and Thompson (2011) reviewed the roles and competencies of online teachers. They concluded that there are five areas of focus for professional development and online teaching practice. Online teachers need PD trainings or modules that create an environment for faculty to:

1. find their teacher-selves as online faculty through reflective questions and challenges to their existing beliefs and practices,

2. be active agents and to be listened to as they engage in a collaborative culture of course design and development,

3. be encouraged to pursue pedagogical inquiry and instructional creativity,

4. engage in learning-centered teaching approaches, and

5. promote community-building around online teaching.

Later, Baran \& Correia (2014) identified three levels of professional development for online teaching that should be considered when designing professional development.

1. Teaching (the PD content): Facilitated by workshops/showcases; training programs for instructional and assessment strategies; and one-on-one assistance

2. Community (the PD environment): Facilitated by collegial learning groups; peer support programs (peer observation and feedback); and mentoring programs

3. Organization (the PD context): Facilitated by rewards and recognitions and by positive organizational culture toward online education in which face-to-face and online instruction are valued and supported.

Implications for PD Design

Therefore, the design of the PD for online teaching aspired to offer two things; (1) an online learning environment that reflected the five opportunities for faculty learning and engagement, and (2) the integration of all three levels of PD. The resulting design of the learning environment was conceptualized with:

1. a focus on teaching to support instructors' understanding of the technology, theories of learning, and real-world application of instructional strategies and PD content,

2. usage of the existing platforms for online instructional delivery to model the student experience both pro and con to establish a collaborative culture, and

3. empowerment of online instructors to pursue creativity in a learning community environment supportive of exploration and experimentation.

\section{For Content Design for Online Education Professional Development}

The goal of the WGU/CUNY professional development series was to support full- and part-time faculty with relevant tools as they continued to advance their pedagogical skills in online settings. The series was not intended to be an introduction to Learning Management Systems (LMS), Blackboard, etc. The intention 
was to create an understanding of effective online education practices that showed evidence of making a positive difference in student learning and success.

Through surveying students' satisfaction and perceived learning, Swan (2001) identified ten concepts that support effective design of online courses. Swan further distilled those ten concepts into three design factors that contribute to the success of online courses, features that have withstood the test of time through nearly 20 years of subsequent practice and the maturation of online teaching and learning:

1. clear and consistent structure of the course itself (micro-modular, embedded, and retention driven),

2. frequent and constructive interaction with a course instructor (learner-centric and varied approaches), and

3. valued and dynamic discussion (learning community).

Regarding student engagement in learning, Garrison and Cleveland-Innes (2005) explored the issue of how to facilitate cognitive presence in online learning. Their findings underscored the importance of and the need for dynamic discussion for deep learning to occur in an online setting. They recommended that facilitating online discussion should have clear participation requirements in terms of length, content expectations, and timeliness. Critical components of discussion facilitation included posing engaging questions, keeping discussion focused, testing and challenging ideas, modelling contributions, and monitoring student progress in creating meaning, confirming understanding, and encouraging participation.

Probing characteristics that students considered essential to effective online teaching, Young (2006) found that the most highly correlated instructor-centered characteristics for effective online instruction were:

1. adapting to student needs by being flexible but demanding high quality work,

2. using meaningful examples,

3. motivating student to do their best by staying on task to achieve learning goals,

4. facilitating effectively through well-designed discussion and instructor involvement,

5. valuing the course by demanding high quality work, posting a thorough syllabus at the start of the course,

6. communicating effectively by consistent and timely responses and providing quality feedback, and

7. being concerned about student learning by setting expectations that were clear, fair, and challenging.

\section{Implications for Module Content}

Although a variety of course design factors and instructor characteristics support effective online teaching and learning, the most effective strategies for student engagement in an online setting appear to be related to facilitating interaction among students and ideas. Therefore, the PD content design included a focus on the ways that online course design and instructor interventions can engage students through high quality discussion, effective facilitation, and providing meaningful feedback. In the final version of the trainings, instructional strategies were highlighted, not as a "bag of tricks" or "hot tips" but as part of an integrated system of student support that includes design, student engagement, and student learning and success within a learning community. As will be explored in a later section, factors specific to the training in terms of size of the cohort, time of year, incentives, and criteria for recruitment proved to be as critical to consider for faculty professional development as were the theoretical underpinnings.

\section{OVERVIEW OF THE CUNY/WGU COLLABORATION}

Each CUNY college has its own unique culture and faculty within academic departments at each college that determine the milestones required to achieve tenure and promotion. While there is a degree of centralized guidance and structure at CUNY, the specifics of faculty incentives are established at the individual college or department level. However, if the CUNY system values and prioritizes excellence in teaching, then, as a system, CUNY must be willing and able to incentivize, recognize, and reward good 
teaching. This can be done through an examination and revision of the teacher/scholar incentive and reward structure through, amidst other initiatives, a structured and goal-driven dialogue that engages the various colleges and schools that make up CUNY.

In 2019, when Felix Matos-Rodriguez became CUNY Chancellor, he established a priority on teaching and pedagogy. System leaders immediately began discussions focused on an approach to open the conversation to design an incentive structure to build enthusiasm and innovation for excellence in teaching. Importantly, no system bylaws address the incentive structure, so no systemwide policy change was required. What was needed was culture change across 25 unique institutions that comprise the federated system serving the City of New York.

Like other colleges and universities worldwide at the outset of the COVID pandemic, CUNY's fulland part-time faculty and students were suddenly forced into online teaching and learning in the midst of the Spring 2020 semester. Across CUNY's 25 colleges and professional schools, some faculty were masterful at using the CUNY LMS, Blackboard. However, many had little experience engaging students in online learning settings and needed support as they shifted their teaching practice.

Full- and part-time faculty members at CUNY felt immense stress and worry about letting their students down, along with concerns that students would drop their classes. Additionally, faculty were seeking the tools to adapt to the COVID reality of online teaching and re-establish control and direction over their curriculum, beyond simply placing important materials on their respective LMS.

CUNY responded to the urgent needs through the development and delivery of a four-week session in April, Online Teaching Essentials, enrolling 2,500 faculty members across four cohorts from May to August. In addition to this workshop, faculty expressed the need for continued support beyond the basics, including pedagogy, student engagement, and fostering interactivity in online settings.

CUNY's innovative pedagogy team offered a series of seminars on mindset, open pedagogy, academic quality, and Helping Bring the Classroom to the Screen, the collaboration with WGU. CUNY supported an additional 2,100 faculty members through these four advanced opportunities, bringing the total to more than 5,000 full- and part-time faculty members across the system who engaged in development opportunities over the summer.

To energize a culture shift to incentivize excellence in teaching and pedagogical innovation, CUNY will establish a Blue Ribbon Task Force of primarily faculty with some administrator participation to begin a conversation on what a recalibrated teacher/scholar incentive structure would look like. The goal of the task force is to create a venue and a format for serious conversations across the university through a shared governance model. The University Faculty Senate will work in collaboration with CUNY to identify faculty members to serve on the task force, which will host open panels, colloquia, and discussions across the CUNY system as well as in individual colleges and groups of colleges.

\section{Participant Selection}

To ensure that Bringing the Classroom to the Screen would be well-aligned to the needs of participants, faculty were invited who met the following criteria:

- full-time or long-term adjunct faculty who would be teaching in Fall 2020 a minimum of two general education or degree program courses critical to academic momentum and who had some online teaching experience prior to Spring 2020, or

- $\quad$ prior completion of an intro-level Online Teaching Essentials workshop or similar course and an expressed interest in sharing strategies gained with peers and serve as a conduit for resources, or

- instructional support staff such as those who serve in a Center for Teaching and Learning (CTL) role or who support online instruction.

\section{Collaborative Design and Delivery}

Upon receipt of external funding, CUNY and WGU began designing PD with bi-weekly meetings focused on content and logistics. Collaborators introduced, discussed, and prioritized topics with input from WGU personnel, CUNY Central administration, and a CUNY faculty representative. Preliminary 
discussions settled on the need to model design elements using the CUNY-preferred platform and supplementing online content with synchronous events highlighting the four modularized topics through the voices of experienced online instructors from both WGU and CUNY. This hybridized, shared modeling approach was the backbone of the training.

The synchronous sessions kicked off each training module and provided an overview of the module topics in the form of a mini lecture. Guest panelists, one panelist from CUNY and one panelist from WGU, offered insights in response to the module's essential question. The sessions concluded with Q\&A, taking questions and providing live answers from or discussion among the panelists, facilitators, and participants.

The asynchronous learning space for the training was the CUNY LMS (Blackboard) where participants were enrolled as students in the WGU-CUNY Professional Development Training. Participants were able to engage with the content at their own pace and in their own way. There was no right way to approach the modules. The modules were intended to build upon each other and were released sequentially following the synchronous sessions. The training content was designed with materials, resources, discussions, and assessments to deepen participants' knowledge and skills for teaching online. The online course site was also designed to model the principles, best practices, and high-impact strategies as well as to provide the participants with a realistic student experience of the good, the bad, and the ugly of online teaching and learning.

There were four key learning outcomes for the training that, in turn, formed the titles and content of four modules. The Learning Outcomes (LO) were:

a. Facilitate faculty ability to develop high quality courses grounded in effective practices to maximize student success

b. Enhance faculty incorporation of principles of pedagogical design

c. Enrich faculty ability to effectively engage students online

d. Support faculty to effectively guide student research in an online environment

\section{Module 1}

Highly Effective Practices for Student Success explored seven effective practices for online teaching in higher education and related each of the practices to the content of the four modules that comprised the WGU/CUNY Professional Development training in supporting course design and student engagement in an online world. In the synchronous session, invited guests defined effective practices and what, if anything, may be missing from teaching excellence when delivering instruction online. Participants were expected to:

- Identify at least 5 effective practices

- Analyze learning objectives for alignment, support, and measurability

- Critique the clarity of course expectations for student performance

\section{Module 2}

General Principles of Excellence in Pedagogical Design introduced and explored pedagogical principles specific to online courses that mediate the unique challenges and affordances of online teaching and learning. In the synchronous session, two guest speakers addressed this essential question of uniqueness. In the asynchronous learning module, the principle of Establishing Your Online Presence was highlighted. Participants were expected to:

- Align general pedagogical design principles with instructional strategies

- Apply a design principle to a scenario of need

- Evaluate a course for integration of strong design principles

- Create a continuous improvement plan for incorporating a design principle or related instructional strategy 
Module 3

High Impact Discussion Strategies addressed the creation and maintenance of an engaging online environment. During the synchronous session, guest panelists explored this idea while the asynchronous module focused on effective discussion strategies and student engagement in general. Participants were expected to:

- List at least one discussion starter for each type of thinking: convergent, divergent, and evaluative.

- Reword 2 discussion questions for greater impact

- Analyze/critique a rubric for evaluating discussion

- Create an assignment and a rubric for evaluating student group work contributions

\section{Module 4}

Guiding Student Research in an Online Environment was delivered asynchronously without an accompanying synchronous session. Videos included a mini-lecture and an interview with a research instructor. The Blackboard content defined actionable feedback and established how to provide it to students. Participants were expected to:

- Distinguish effective from non-effective feedback.

- Rewrite sample feedback for actionability

- Provide actionable feedback on a sample student research paper

\section{Promoting and Setting the Stage to Scale Up}

Promoting the voluntary opportunity for faculty to participate in Bringing the Classroom to the Screen included multiple strategies. CUNY leaders sent a detailed invitation to a listserv of more than 5,000 faculty members who had participated in professional development offerings throughout spring and summer and met the selection criteria detailed above. The CUNY University Faculty Senate, Faculty governance leaders, and Faculty Advisory Council shared information across the colleges about all the professional development offerings the system was providing. Importantly, in all the communication of the opportunities, the voluntary nature of participation was emphasized, as well as recognition that CUNY recognized the efforts faculty had made to transition to online teaching full-time. Additionally, in recognition of that effort, CUNY committed to a $\$ 500$ stipend for faculty members who opted to participate and complete the workshop.

To scale up the learnings from Bringing the Classroom to the Screen, CUNY invited the Center for Teaching and Learning (CTL) Directors and other instructional support staff from each of its 25 colleges to participate in the training. Their participation built a strong foundation for a train-the-trainer approach, where each CTL Director and instructional support staff can share the model with faculty members at their respective colleges. Finally, we created an Innovative Pedagogy listserv that included all faculty who had participated in the summer professional development trainings over the summer of 2020 to support a community of practice moving forward and encourage further sharing and dissemination of best practices among faculty across the system.

\section{MEASURING AND SUPPORTING SUCCESS OF THE TRAINING AND OF PARTICIPANTS' PRACTICE}

\section{Culminating Project and Reflection}

A culminating project $(\mathrm{CP})$ was a requirement of the training and was designed to provide participants with an opportunity to choose a learned strategy, best practice, or instructional technique to implement in their online courses. The product related to the $\mathrm{CP}$ was two-fold:

1) The first part of the $\mathrm{CP}$ was the submission of an abstract describing the learned strategy, best practice, or technique that the participant intended to implement or implemented as a result of the professional development training. The abstract included the following information: 
a. A description of the learned strategy, best practice, or technique for implementation ("What" the participant intended to do),

b. A rationale for implementation ("Why" the participant chose the strategy, practice or technique), and

c. Success Measure ("How" the participant would know if the strategy, practice, or technique worked).

2) The second part of the $\mathrm{CP}$ was the submission of a reflection upon the professional development training as a whole.

A qualitative analysis of 107 culminating projects clustered a set of findings and insights from faculty work into five areas:

- Affected Courses

- Proposed Online Teaching Strategies, Technique, or Best Practice (STBP)

- Impactful Training Modules

- Inquiry-Based Success

- Participant Motivation

\section{Affected Courses}

Course titles ranged from introductory courses in General Biology and Music Survey to culminating research courses such as Social Science Research Methods and Program Evaluation. Twenty-three (21.5\%) of the 107 culminating projects were submitted without identifying the course title. Of the remaining 84 affected courses, $24(22.4 \%)$ were categorized as general education courses and 60 (56\%) were identified as discipline specific or professional courses.

\section{Proposed Online Teaching Strategy, Technique, or Best Practice}

Online teaching strategies identified for implementation ranged from adding synchronous sessions to exclusively asynchronous courses and offering varied formats to course content delivery. Over 107 strategies were categorized into ten training topics with Student Engagement strategies and the pedagogical design principle of Letting Students Do the Work representing 38.3\% of the proposed strategies.

\section{Impactful Training Modules}

The WGU/CUNY Professional Development Training offered four modules: Module 1: Best Practices for Student Success; Module II: General Principles of Excellence in Pedagogical Design; Module III: High Impact Discussion Strategies; and Module IV: Guiding Student Research. Of the four modules offered during the training, modules I and II were cited as the most impactful with 46 (42.9\%) and 38 (35.5\%) of the participants, respectively, identifying a strategy or strategies from these modules for integration into their online courses.

\section{Inquiry-Based Success}

The measures by which participants would evaluate the successful implementation of their online course strategies ranged from tracking the number of discussion posts to analyzing students' responses to feedback surveys. One hundred and seven success measures were categorized into six areas: raw numbers and percentages of increase/decrease, grades, student feedback, rubric values, comparison with a prior year, and qualitative analysis of reflections, essays, etc. $54.2 \%$ of participants, in equal numbers (29 each), listed either raw numbers/percentages of increase/decrease or qualitative analysis as the way in which they would judge successful implementation of proposed online teaching strategies.

\section{Participant Motivation}

The rationale for participants' decisions about which strategies to implement in their online course(s) were analyzed as being personal, related to training-content, or student-centric. Forty-one (38.3\%) of the 
participants attributed student-centric motivations for their choice of online strategies, such as to increase student understanding of course content, to improve learning, or to develop critical thinking abilities.

\section{Continuous Improvement}

Throughout the summer of 2020, WGU/CUNY Professional Development Training in online teaching, facilitators sought participant feedback through surveys and reflections. This continuous feedback cycle resulted in a set of recommendations for future trainings in the key areas of planning, learner experience, groupwork, point values/grading, and resources/ community.

\section{Planning}

Any professional development training for higher education faculty should consider the need for clarifying the definitions and details of collaboration and support: a shared understanding of and expectations for setting up and refining the asynchronous delivery of training content, reviewing and revising training content to meet faculty needs, sharing workload of grading and providing feedback to participants, and supporting the LMS platform technical glitches and need for modification.

The timing of the training should be collaboratively explored. Through implementation, we found that the content from the fourth module on effective feedback could have been easily integrated into the third module on student engagement, thereby reducing the load on faculty during the beginning of a semester. Also, the summer 2020 PD training was delivered over the course of four weeks. Faculty needed more time to complete the modules and to reflect on the content to integrate enhancements into their courses. Offering the training at regularly scheduled intervals, once every two weeks or so, would accommodate more faculty.

As a final point for continuous improvement during planning, the capacity for the training should be considered. In the summer of 2020, there were over 300 participants. As a result, facilitators were pressed to provide individualized and personalized feedback.

Upon reflection on the summer series and in light of the continued need for training, in the winter of 2021, the training was offered again implementing improvements suggested by the first workshop's assessment. CUNY had funds to support a small cohort to implement and pilot the changes made as a result of the assessment. During this offering, 40 faculty were selected. The 40 participants were further subdivided into two equal groups roughly based on their area of teaching. Each group was led by one of the same two WGU and CUNY facilitators. The reduction in the number of participants resulted in the following outcomes and learnings:

1. Less is more. Limiting the cohort to fewer participants lent itself to personalization, more learning, increased engagement, and fewer dropouts.

2. Having an actively engaged co-facilitator from CUNY created opportunities for heightened institutional and personal support as well as time management.

3. Participants were more likely to engage (through emails, office hours, etc.) with an institutionally affiliated co-facilitator than a partner entity.

4. Continuous improvement does not mean that everything is perfect; it means that many things are improved and other opportunities emerge.

5. Teaching is teaching: adjust, adapt, learn, improve, repeat.

\section{Learner Experience}

One of the most surprising aspects of Bringing the Classroom to the Screen was the diverse reaction of participants to the training itself. Some felt the content was too simplistic while others felt it was perfect for them. Some felt the organization was frustrating while others felt the course was well organized and well delivered. Some engaged deeply with additional readings and took the training as an opportunity to improve their own course before the fall semester while others seemingly engaged with a "do-the-leastpossible" and "get-this-over-with" mentality. As these varied reactions surfaced, the training adjusted and focused more on how the experience itself was a learning opportunity. Many participants openly commented that they were feeling the same range of emotions and experiences that their own students must 
have felt taking online classes This was an ancillary benefit and welcome response to the training, as more faculty were able to experience first-hand what their students experience.

If this type of professional development training for higher education faculty were replicated, awareness of the intended learner experience should be retained and made more salient. However, communication to potential participants should identify the training as experiential without foreshadowing the learner experience to the extent that it becomes inauthentic.

\section{Groupwork}

From the very first module, randomly assigned groups were available to all participants. Discussion, email, file exchange, and the ability to host group meetings were enabled. However, no one took advantage of the group option when it was not an assigned activity. This was surprising, particularly when (by module 2) participants were asking for groups to better communicate in discussions, etc. However, it also reflected the exhaustion many faculty members experienced with the transition to online teaching. When offering similar professional development opportunities in response to unexpected challenges, training developers should acknowledge the effects of transition fatigue.

In response to these participants' requests, groups were redeveloped, randomly assigned, and implemented for entering into the discussion topic for Module 3. A few participants commented upon their "enjoyment" of the smaller group venues. Some participants indicated that they had "bonded" with other participants and wished they could have selected their own group members. Other participants suggested that discipline-alike groups should have been formed. Still other participants said it was too little, too late and a distraction. During the second iteration of this workshop, groups were implemented from the very beginning with discipline-alike groupings: teacher education faculty together, lab sciences together, etc. Group members got to know each other and engaged frequently through multiple discussions.

\section{Point Values/Grading}

Originally, the training plan required only the submission of a culminating project as evidence of training completion. However, as the planning evolved, a point value system was adopted (consistent with other CUNY-led trainings) and a total point vs. required number of points for training completion was determined. The addition of a point system brought with it the usual difficulty of assigning points to work: specifying point values using consistently applied rubrics and dealing with appeals for point deductions. Many participants commented that the points and discussions around them reminded them of their own students requesting grade appeals. While the facilitators assured the participants that small point deductions, which are expected as someone learns new material, would not derail their passing grade, many participants still expressed angst over the possibility. If this training were replicated, an alternative to point values could be beneficial with a focus on participant requirements and tracking completion/non-completion only.

\section{Resources and Community}

A variety of resources were included in the training to balance practical and theoretical resources, to provide a variety of formats (video, reading, blog, etc.), and to highlight resources related to the content specific to the module topic. The resources for each module were intended to be foundational and worksin-progress with new resources added, updated, and contributed by participants. Participants requested access to these shared resources beyond the life of the training. Both CUNY and WGU have institutional priorities and support for OER (Open Educational Resources). For that reason, the training resources were migrated to a repository that is accessible to graduates and participants beyond the life of the training.

During the training, participants developed an informal, fledgling community of practice. They began to seek out each other for tips and proven practices as well as to ask for advice and to share experiences and interests. Some participants expressed the desire for the developing community to continue beyond the life of the training. Continuing the sharing opportunity for graduates should also be considered. 


\section{CULTURE CHANGE TO INCENTIVIZE EXCELLENCE IN TEACHING}

Importantly, because faculty incentive structures for tenure, promotion, and re-appointment are not specified in CUNY's system bylaws, the recalibration will largely be a culture change on each campus, with the potential for minor adjustments to each college's governing documents to reflect this change. The size and scope of the system means that CUNY is an amalgam of different cultures. As such, the University Faculty Senates, the various colleges' Senates, college administrators, and faculty in the academic departments will be key to bringing about meaningful culture change that honors the historic practices in place for promotion and tenure milestones at each college in the system.

There may be substantive changes at many campuses with the inclusion of incentives in the reward structure for good pedagogy and good teaching. It will be critical to embed support for faculty in these aspects of their professional practice.

\section{NEXT STEPS}

In moving forward from the pandemic, which created a scenario in which faculty had little control over their academic lives, it will be critical to support faculty in ways that help them move from a feeling of being buffeted by forces outside their control to once again having autonomy and direction over their pedagogy. Providing faculty with tools to re-assert their own individual specializations and personalities in the classroom will be essential to that process. To be more successful, faculty need to be equipped with a full array of tools to support teaching and learning in all environments - traditional face-to-face, hybrid, synchronous, asynchronous, and others.

Faculty and administrators cannot unlearn the lessons garnered during the pandemic. The lessons may play out in many different wants. For example, some faculty may never again collect a piece of paper from a student or expect that all their students will be in the same room at the same time.

For system administration, it is critical to support faculty in ways that are helpful and aligned with faculty visions for teaching. For CUNY, the faculty development offerings in the pandemic actually overcame a previous barrier the system faced-bringing faculty from different campuses together in a central location for professional development. Through its online offerings in 2020 and 2021, CUNY was able to remove geographic barriers to faculty participation in system-wide offerings.

An additional barrier to faculty participation is the need to provide faculty with financial incentives to complete professional development offerings during the summer. Throughout the academic year, faculty would be more willing and likely to engage and commit to ongoing training and development to improve and enhance their teaching if such participation is built into the tenure and promotion reward structure. CUNY is committed to funding its top priorities, which include a focus on excellence in teaching. The system's budget request to the State of New York included substantial support for CUNY's Innovative Teaching Academy and faculty stipends to provide the funds needed to support this stated priority. Further, the system has garnered significant grant support for faculty development activities, including a substantial grant from the Andrew W. Mellon Foundation.

In conclusion, the collaborative professional development training for faculty engaging in online teaching was a positive learning experience. Lessons learned informed the ways forward with strong potential for replication and for broadening the impact on policies and practices related to good teaching in higher education.

\section{ACKNOWLEDGEMENT}

Funded in part by the Bill and Melinda Gates Foundation. 


\section{REFERENCES}

Baran, E., \& Correia, A.P. (2014). A professional development framework for online teaching. Tech Trends, 58(5), 96-102. DOI: 10.1007/S11528-014-0791-0

Baran, E., Correia, A.P., \& Thompson, A. (2011). Transforming Online Teaching Practice: Critical Analysis of the Literature on the Roles and Competencies of Online Teachers. Distance Education, 32(3), 421-439.

Garrison, D.R., \& Cleveland-Innes, M. (2005). Facilitating cognitive presence in online learning: Interaction in not enough. The American Journal of Distance Education, 19(3), 133-148.

Picciano, A.G. (2017). Theories and frameworks for online education: Seeking an integrated model. Online Learning, 21(3), 166-190.

Swan, K. (2001). Virtual interaction: Design factors affecting student satisfaction and perceived learning in asynchronous online courses. Distance Education, 22(2), 306-331.

Young, S. (2006). Student views of effective online teaching in higher education. The American Journal of Distance Education, 20(2), 65-77. 